\title{
Acute consumption of phenolic-rich olive leaf extract reduces arterial stiffness and decreases interleukin-8 production
}

\author{
S. Lockyer, P. Yaqoob, J. P. E. Spencer and I. Rowland \\ Department of Food and Nutritional Sciences, University of Reading, RG6 6AP, UK
}

There is an association between the high consumption of olive oil, the principle fat in the Mediterranean diet, and a reduction in chronic disease risk $^{(1)}$. Data has highlighted the health-promoting effects of phenolic compounds present within the water-soluble fraction of the $\mathrm{oil}^{(2)}$. However, olive leaves are the richest source of phenolics within the olive plant (Olea europaea), with the secoiridoid, oleuropein, being the most abundant compound.

In a randomised, controlled crossover clinical trial, 18 subjects ( 9 male, 9 female) aged 19-40 years consumed either 4 phenolic-rich olive leaf extract (OLE) capsules, (each containing $400 \mathrm{mg}$ olive leaf extract, equivalent to $2.4 \mathrm{~g}$ fresh olive leaf and $14.53 \mathrm{mg}$ oleuropein, plus $672.5 \mathrm{mg}$ safflower oil) or a control ( $900 \mathrm{mg}$ safflower oil) in a random order, separated by a 4-week washout. Blood and urine were collected to ascertain the bioavailability of OLE (data not shown). Subjects consumed a low polyphenol diet for 24 hours prior to each study day. Digital volume pulse-derived stiffness index (DVP-SI) was obtained via PCA2 Pulse Trace at baseline and 30, 60, 90, 120, 180, 240, 360 and 480 minutes after capsule ingestion. A low fat, low polyphenol meal was provided after the 240 minute sample. For whole blood cytokine production, blood collected at baseline, 60, 180 and 360 minutes was diluted 1:1 with RPMI 1640 medium (containing $1 \%$ antibiotics), stimulated with LPS $(1 \mu \mathrm{g} / \mathrm{ml})$ and incubated for 24 hours $\left(37{ }^{\circ} \mathrm{C} ; 5 \% \mathrm{CO} 2\right)$. Supernatants were collected and stored at $-80{ }^{\circ} \mathrm{C}$ until analysis for interleukin(IL)-1 $\beta$, IL-6, tumour necrosis factor(TNF)- $\alpha$, IL-8 and IL-10 using Fluorokine MAP ELISA kits and a Luminex reader. Prism (GraphPad software, USA) was used to analyse the data. Differences in DVP-SI, IL- $1 \beta$, IL-6, TNF- $\alpha$, IL- 8 and IL-10 by treatment were identified using a two-way ANOVA with repeated measures. Post hoc analysis was subjected to Bonferroni correction. $\mathrm{P}$ values less than 0.05 were treated as significant.

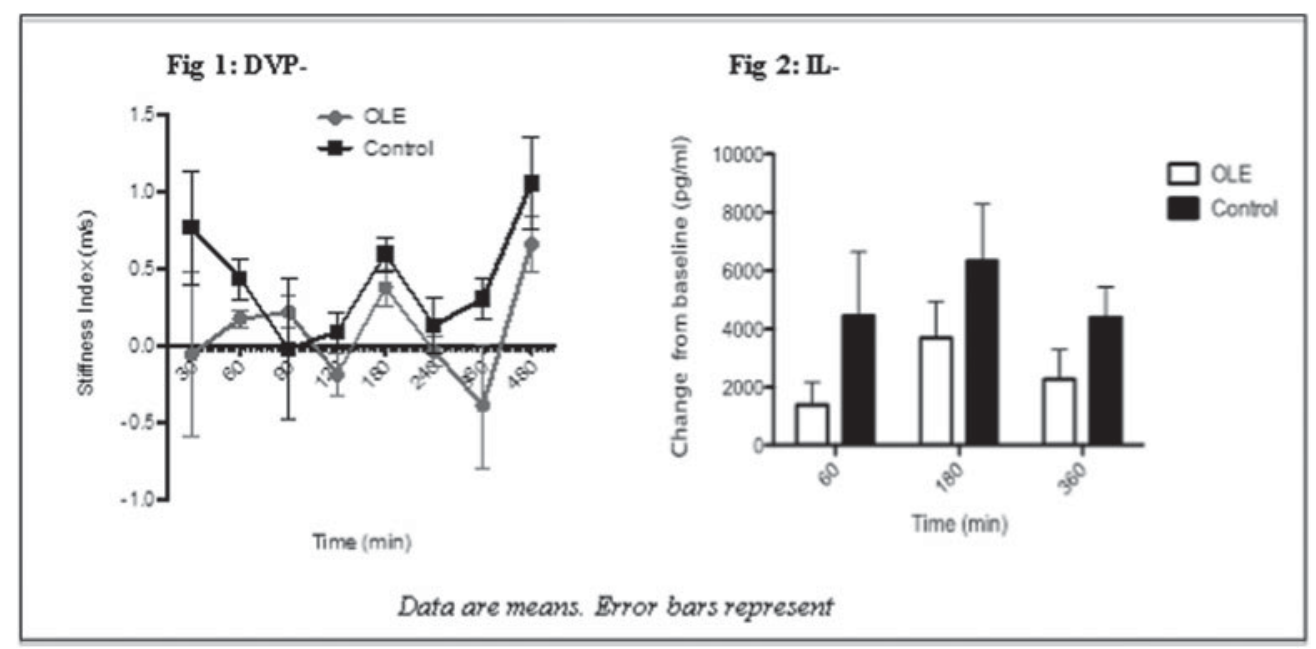

DVP-SI and IL-8 were significantly lower over the course of the study day $(P<0.01$ and $P<0.05$ respectively) when subjects consumed OLE capsules $v s$. the control. Post hoc analysis revealed no significant differences at any individual time point over the day. There was no significant treatment effect on IL-1 $\beta$, IL-6, TNF- $\alpha$ or IL-10.

The effect of OLE phenolics on vascular function, an important CVD risk marker, has not been studied previously. The current study demonstrated that OLE phenolics have anti-inflammatory properties during the postprandial period, and decrease arterial stiffness. Further work aims to characterize the mechanisms underlying these effects.

1. López-Miranda J, Pérez-Jiménez F, Ros E, et al. Nutr Metab Cardiovasc Dis. 2010; 20(4): 284-94.

2. Yang D-P, Kong D-X, Zhang H-Y. Food Chemistry. 2007; 104(3): 1269-71.

3. Ruano J, Lopez-Miranda J, Fuentes F, et al. J Am Coll Cardiol. 2005; 46(10): 1864-8. 